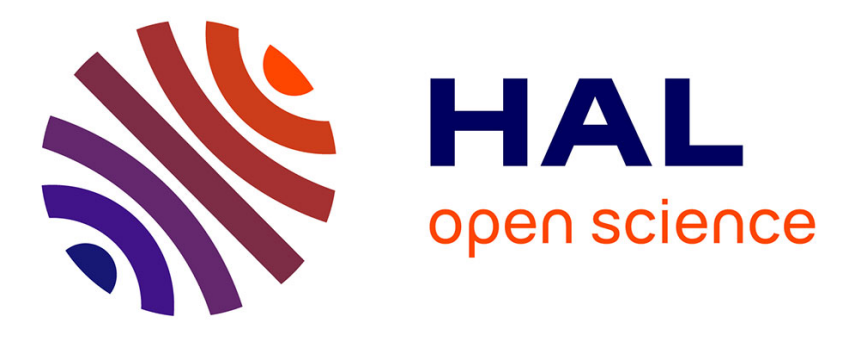

\title{
Search-Based Testing for Embedded Telecom Software with Complex Input Structures
}

\author{
Kivanc Doganay, Sigrid Eldh, Wasif Afzal, Markus Bohlin
}

\section{To cite this version:}

Kivanc Doganay, Sigrid Eldh, Wasif Afzal, Markus Bohlin. Search-Based Testing for Embedded Telecom Software with Complex Input Structures. 26th IFIP International Conference on Testing Software and Systems (ICTSS), Sep 2014, Madrid, Spain. pp.205-210, 10.1007/978-3-662-448571_17. hal-01405289

\author{
HAL Id: hal-01405289 \\ https://hal.inria.fr/hal-01405289
}

Submitted on 29 Nov 2016

HAL is a multi-disciplinary open access archive for the deposit and dissemination of scientific research documents, whether they are published or not. The documents may come from teaching and research institutions in France or abroad, or from public or private research centers.
L'archive ouverte pluridisciplinaire HAL, est destinée au dépôt et à la diffusion de documents scientifiques de niveau recherche, publiés ou non, émanant des établissements d'enseignement et de recherche français ou étrangers, des laboratoires publics ou privés.

\section{(c)(1)}

Distributed under a Creative Commons Attribution| 4.0 International License 


\title{
Search-based Testing for Embedded Telecom Software with Complex Input Structures
}

\author{
Kivanc Doganay ${ }^{1,2}$, Sigrid Eldh ${ }^{3,4}$, Wasif $\mathrm{Afzal}^{2}$, and Markus Bohlin ${ }^{1,2}$ \\ 1 SICS Swedish ICT AB, Kista, Sweden \\ 2 Mälardalen University, Västerås, Sweden \\ ${ }^{3}$ Ericsson AB, Kista, Sweden \\ ${ }^{4}$ Karlstad University, Karlstad, Sweden
}

\begin{abstract}
In this paper, we discuss the application of search-based software testing techniques for unit level testing of a real-world telecommunication middleware at Ericsson. Our current implementation analyzes the existing test cases to handle non-trivial variables such as uninitialized pointers, and to discover any setup code that needs to run before the actual test case, such as setting global system parameters. Hill climbing (HC) and $(1+1)$ evolutionary algorithm (EA) metaheuristic search algorithms are used to generate input data for branch coverage. We compare $\mathrm{HC},(1+1) \mathrm{EA}$, and random search with respect to effectiveness, measured as branch coverage, and efficiency, measured as number of executions needed. Difficulties arising from the specialized execution environment and the adaptations for handling these problems are also discussed.
\end{abstract}

\section{Introduction}

Embedded systems are prevalent in many industries such as avionics, railways and telecommunication systems. The use of specialized microprocessors such as digital signal processors (DSPs) and electronic control units have enabled more complex software in the embedded domain. As a consequence, quality control has become more time consuming and costly, similar to the non-embedded software domains.

It is well known that software testing is an expensive activity [6]. Thus considerable research has focused on automating different test activities, notably software test data generation. In recent years, the use of metaheuristic search algorithms have shown promise in automating parts of software testing efforts [3], including test data generation, which is commonly referred to as search-based software testing (SBST). SBST has received increasing attention in the academia. While random testing and fuzzing have gained reasonable visibility and acceptance, search-based approaches are not yet adopted in the industry. To gain wider acceptance, we believe that experiments of search-based techniques on real-world industrial software should be performed. With a family of such experiments, we would be in a better position to argue for the industrial uptake of SBST.

In this paper, we present a case study of applying search-based testing on a real-world telecommunication middleware at Ericsson. We use hill climbing 
(HC) and $(1+1)$ evolutionary algorithm (EA) metaheuristic search algorithms to generate unit level input data that exercise different branches in the control flow. The algorithms, along with random search as a baseline, are compared for effectiveness, measured in branch coverage, and efficiency, measured in number of executions. Existing test cases are automatically analyzed in order to handle complex data structures, pointers, and global system parameters. We also discuss difficulties arising from the specialized execution environment, and the adaptations for handling these problems. A further detailed account of the case study can be found in the corresponding technical report [1].

\section{System Under Test}

The system under test is written in the DSP-C programming language. DSP-C is an extension of the $\mathrm{C}$ language with additional types and memory quantifiers designed to allow programs to utilize hardware architectural features of DSPs [2]. Ericsson uses a proprietary compiler to compile DSP-C code for various DSP architectures, and a proprietary simulator to execute the resulting binaries on a normal Linux environment. The whole tool chain is connected via a test execution framework. Currently we do not integrate to the test framework, but we extract certain information from it.

\subsection{Analysis and Instrumentation}

We adapted pycparser ${ }^{5}$, which produces the abstract syntax tree (AST) for the $\mathrm{C}$ language, to the DSP-C language including Ericsson specific memory identifiers. Our tool analyzes the resulting AST to produce the control flow graph (CFG) of the function under test (FUT).

The Assimilator module (Fig. 1) instruments the FUT by inserting observation code at the branching points in the CFG. The code is instrumented without changing its functional behavior. For example, the statement if $(a>b \quad \& \& \quad c)$ is instrumented as if (observe_gt $(12,0, a, b)$ \&\& observe $(12,1, c)$ ), where 12 is the branch identifier in the CFG, while 0 and 1 are the clause identifiers in the given expression. Note that the variable $c$ will not be read if the first condition $(a>b)$ is false. This ensures that instrumentation do not change the functional behavior of the FUT.

Existing test cases. We analyze the existing test cases (developed by Ericsson engineers) for a given FUT to discover the input interface, and any setup code that needs to be executed before calling the FUT. Test cases may include some setup code, such as allocating memory, or setting a global variable. We replicate these setup code sections in the generated template test case. Then all assignments in the test code are parsed, and the discovered assignments are used to define the input space. Only the variables and fields of data structures which are set in at

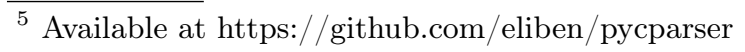




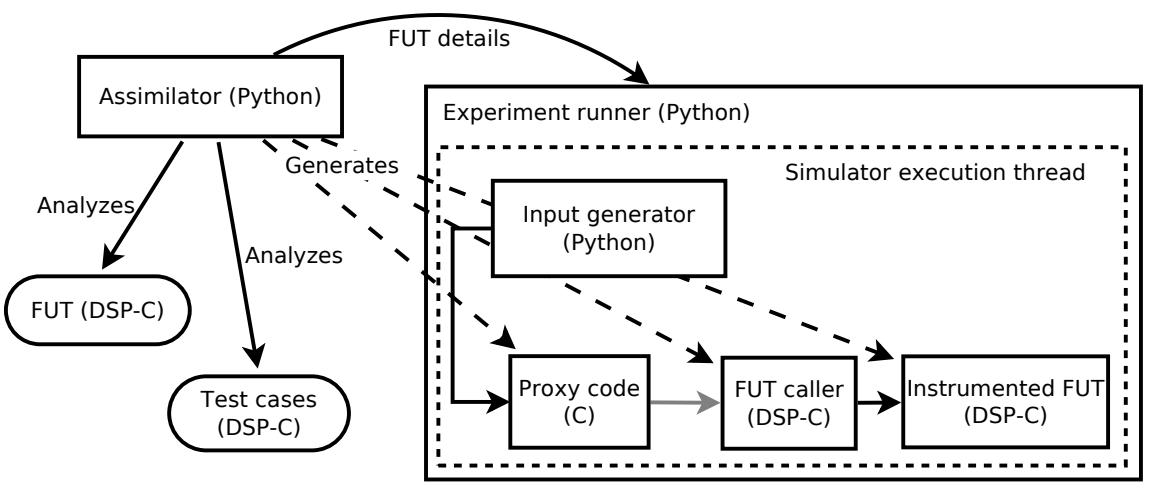

Fig. 1. Prototype's architecture and the execution environment.

least one test function are used to construct the input space, as it is likely that variables and fields which are never set are uninteresting for testing purposes. In particular, this is true for variables which do not affect the execution of the FUT (e.g., output variables), or for variables which are initialized in the setup phase and should not be changed due to the logic of the system.

\subsection{Execution Environment}

The DSP simulator supports interacting with simulated code in a restricted manner, e.g., direct function calls are not possible. Therefore our implementation was heavily adapted to the simulator.

The Input generator (Fig. 1) implements the search algorithms using a vector representation of basic types, which is oblivious to the actual input structures. The Proxy code and FUT caller (automatically synthesized by the Assimilator module) packs the input vector into the input data structures, which is then passed to the simulated DSP-C environment. After each FUT execution observation data is read to calculate the fitness value of the last input vector. Then the execution cycle is repeated with the next input vector generated by the search algorithm.

\section{Experimental Evaluation}

In this section we compare the experimental results of hill climbing (HC), $(1+1)$ EA, and random search $(\mathrm{RS})$ algorithms for branch coverage on a set of four functions in a library sub-module of Ericsson's middleware code base. Both HC and $(1+1)$ EA algorithms use the same popular fitness function for branch coverage that combines approach level and branch distance [5].

The particular sub-module was selected as it was considered suitable to be tested using the simulator, rather than the real hardware. In particular, suitable sub-modules should have no or minimal inter-module dependencies, and should be 
without timing critical requirements. In this sub-module, functions with the most lines of code and branching statements were selected. The chosen FUTs are listed in Table 1 with structural information. Func_A, Func_B, and Func_C are directly tested by the existing unit tests. However, Func_subA is not executed directly but through Func_A, which we mimic in our test data generation approach. Therefore, Func_A and Func_subA share the same input vector.

Table 1. Structural information of the functions that are included in our study.

\begin{tabular}{lrrrr}
\hline Function & \multirow{2}{*}{$\begin{array}{c}\text { Number of } \\
\text { under test }\end{array}$} & \multicolumn{2}{c}{$\begin{array}{c}\text { Input vector size } \\
\text { branches }\end{array}$} & \multicolumn{2}{c}{ Original Reduced } \\
\hline Func_A & 191 & 20 & 508 & 30 \\
Func_subA & 37 & 6 & 508 & 30 \\
Func_B & 352 & 20 & 143 & 28 \\
Func_C & 179 & 32 & 659 & 146 \\
\hline
\end{tabular}

Experiments were run on Ericsson servers using the simulator. We use execution count (i.e., the number of calls to the FUT) for comparison, instead of the clock time, so that the efficiency of our implementation, interactions with the simulator, or the server load do not affect the results. For practical purposes we imposed a limit of 1000 executions (FUT calls) per targeted branch. In order to account for the stochastic nature of the search algorithms, we repeated each experiment 50 times per algorithm per FUT. The total time for executing all experiments was around 150 hours, or 6 days.

Statistical significance tests and effect size measurements are applied for pairwise comparison of algorithms, with respect to branch coverage and execution counts. We omit this analysis for the sake of brevity, which can be found in the technical report [1]. The minimum, maximum, mean, and median values for the achieved branch coverage by each algorithm are shown in Table 2. Branch coverage of the existing test cases are also listed for comparison. For Func_A and Func_subA all algorithms were able to reach full coverage at most of the runs, while some branches of Func_B were covered only by the HC algorithm.

Table 3 shows the number of executions needed per algorithm for each FUT. Again the minimum, mean, median, and maximum values among the 50 independent runs are reported in the table.

The results indicate that both $\mathrm{HC},(1+1) \mathrm{EA}$, and RS were able to achieve high branch coverage most of the time (Table 2). At least one of the algorithms were able to achieve $100 \%$ branch coverage in the case of three out of the four FUTs. For two of the FUTs (Func_subA and Func_B) the achieved branch coverage was higher than branch coverage of the existing test cases. Therefore, we were able to increase the branch coverage for the FUTs that are studied in our case study. However, search algorithms did not always reach the highest possible branch coverage (Func_C in Table 2).

We observed that RS was not much worse than other algorithms at branch coverage, except for Func_B where HC was the clear winner. This indicates that 
Table 2. Branch coverage achieved by hill climbing, $(1+1) \mathrm{EA}$, and random search algorithms, as well as the existing test cases.

\begin{tabular}{|c|c|c|c|c|c|c|c|c|c|c|c|}
\hline \multirow{2}{*}{ FUT } & \multirow{2}{*}{ Exist. } & \multicolumn{3}{|c|}{ Hill Climbing } & \multicolumn{4}{|c|}{$(1+1) \mathrm{EA}$} & \multicolumn{3}{|c|}{ Random Search } \\
\hline & & $\min$ & mean med. & $\max$ & $\min$ & 1 mean & med. & $\max$ & $\min$ & mean med. & $\max$ \\
\hline Func_A & 1.0 & 0.95 & 0.9961 .0 & 1.0 & 1.0 & 1.0 & 1.0 & 1.0 & 1.0 & 1.0 & 1.0 \\
\hline Func_subA & 0.83 & 0.5 & $0.99 \quad 1.0$ & 1.0 & 0.5 & 0.983 & 1.0 & 1.0 & 0.5 & 0.9471 .0 & 1.0 \\
\hline Func_B & 0.85 & 0.95 & $0.998 \quad 1.0$ & 1.0 & 0.7 & 0.701 & 0.70 & 0.75 & 0.7 & $0.705 \quad 0.70$ & 0.75 \\
\hline Func_C & 1.0 & 0.656 & $0.746 \quad 0.75$ & 0.812 & 0.75 & 50.812 & 0.812 & 0.875 & 0.75 & 0.8240 .812 & 0.906 \\
\hline
\end{tabular}

Table 3. Number of executions (fitness evaluations or FUT calls) that each search algorithm needed before terminating.

\begin{tabular}{|c|c|c|c|c|c|c|c|c|}
\hline \multirow{2}{*}{ FUT } & \multicolumn{4}{|c|}{ Hill Climbing } & \multicolumn{3}{|c|}{$(1+1) \mathrm{EA}$} & \multirow{2}{*}{$\begin{array}{c}\text { Random Search } \\
\text { min mean med. max }\end{array}$} \\
\hline & $\min$ & mean & med. & $\max$ & $\min$ & mean med. & $\max$ & \\
\hline Func_A & 330 & 641 & 576 & 1555 & 288 & 551506 & 1059 & $\begin{array}{llll}73 & 151 & 150 & 310\end{array}$ \\
\hline Func_subA & 167 & 448 & 382 & 3234 & 110 & $493 \quad 408$ & 3143 & $436 \quad 1313034$ \\
\hline Func_B & 1853 & 2231 & 2194 & 2838 & 5650 & 64016364 & 6955 & $53825996 \quad 60426070$ \\
\hline Func_C & 10043 & 11722 & 11551 & 13763 & 7710 & 93969256 & 11970 & $\begin{array}{lll}5605 & 735375919686\end{array}$ \\
\hline
\end{tabular}

many of the branches are relatively easy to cover. One such branch predicate that we found to be common in the code base is inequality comparison with a signed input value and a small constant, such as $(x \leq 10)$. For example, a signed 32-bit integer input $x$ would mean that the probability of a random input leading to the false and true branches approximately equal $(P($ false $) \approx P($ true $) \approx 0.5)$. Similar situation can be observed if the variable being compared is unsigned type, but is defined as a bit field with, e.g., 4-bit length. In embedded software bit fields are commonly used, in order to reduce the memory usage as much as possible.

Furthermore, on average RS is faster (i.e., needs less number of FUT calls) to cover a branch, if it can, compared to other algorithms (Table 3). It is known in the literature that RS is typically faster at covering easy branches than more informed search algorithms.

\subsection{Threats to Validity}

Due to practical reasons (such as computational resources) we imposed arbitrary limits on the execution of the algorithms on each targeted branch. Different execution limits might have led to differing results.

We selected limited number of FUTs from one sub-module. FUTs were selected among the functions with more lines of code and number of branches. We do not know if many smaller FUTs, instead of few big ones, would give significantly different results. In the future, we plan to extend this work to more sub-modules and functions in the code base. 


\section{Discussion and Conclusion}

In this paper, we discussed a case study on application of SBST techniques for an industrial embedded software. The implemented tool was heavily adapted to the simulated execution environment (Section 2). This can be seen as a special version of the execution environment problem, which usually refers to the concerns about interacting with the file system, the network, or a database in the context of non-embedded systems [4]. Moreover, we did not have enough detailed technical knowledge of the system under test to understand the input space. To overcome this problem, we used existing test cases to automatically craft a test case template (Section 2.1).

During the experiments we were able to increase the total branch coverage of existing test cases. So we can say that the employed SBST techniques indicate beneficial results. However, we observed that randomly generated inputs were as effective as more informed search algorithms in many (but not all) cases, which indicate that there are many branches that are easy to cover. A practitioner may prefer to start with random search to cover easy branches first, before using informed search algorithms to target other branches.

For future work, we would like to extend the case study to more FUTs in a similar code base. We plan to investigate alternative ways of interacting with the simulator to reduce the execution times, which was a practical bottleneck for the experiments. Another interesting idea is the use of hybrid search algorithms that can cover easy branches swiftly (e.g., by using random search) and then move to other branches.

Acknowledgments This work was supported by VINNOVA grant 2011-01377, and the Knowledge Foundation (KKS), Sweden. We would like to thank Jonas Allander, Catrin Granbom, John Nilsson, and Andreas Ermedahl at Ericsson AB for their help in enabling the case study.

\section{References}

1. Doganay, K., Eldh, S., Afzal, W., Bohlin, M.: Search-based testing for embedded telecommunication software with complex input structures: An industrial case study. Tech. Rep. 5692, SICS Swedish ICT (July 2014)

2. Leary, K., Waddington, W.: DSP/C: a standard high level language for DSP and numeric processing. In: International Conference on Acoustics, Speech, and Signal Processing, 1990. ICASSP-90. pp. 1065-1068 vol.2 (1990)

3. McMinn, P.: Search-based software test data generation: A survey. Software Testing, Verification and Reliability 14(2), 105-156 (2004)

4. McMinn, P.: Search-based software testing: Past, present and future. p. 153-163. ICSTW '11, IEEE Computer Society, Washington, DC, USA (2011)

5. Wegener, J., Baresel, A., Sthamer, H.: Evolutionary test environment for automatic structural testing. Information and Software Technology 43(14), $841-854$ (2001)

6. Yang, B., Hu, H., Jia, L.: A study of uncertainty in software cost and its impact on optimal software release time. IEEE Transactions on Software Engineering 34(6), $813-825$ (2008) 